\title{
CUIDAR DESDE UNA PERSPECTIVA CULTURAL
}

\author{
Juan Ignacio Valle Racero
}

Enfermero y Licenciado en Antropología

\section{RESUMEN}

En este trabajo, el autor describe los cuidados de enfermeria desde una perspectiva cultural, mostrando particular interés en la relación interpersonal que establecemos con el paciente. Según Valle, la nueva cosmovisión del cuidado debe de contemplar los aspectos socioculturales, los valores, las creencias, los modos de vida, las distintas concepciones de salud y enfermedad, en suma... la diversidad cultural.

Palabras clave: cultural care, antropologia.

\section{CARING FROM A CULTURAL PERSPECTIVE}

\section{SUMMARY}

In this work, the author describes the nursing cares from a cultural perspective, showing particular interest in the interpersonal relation that we establish with the patients. According to Valley, the new cosmovisión of the care must contemplate the sociocultural aspects, values, beliefs, life ways, and the different conceptions from health and disease, in sum... the cultural diversity.

Keywords: cultural care, anthropology.

\section{INTRODUCCIÓN}

El cuidado y su presencia ancestral en la historia de la humanidad es un hecho incuestionado, contrastado y estudiado por multitud de investigadores sociales e historiadores de la enfermería. El cuidado como término polisémico y cargado de conocimiento nos abre las puertas a un fenómeno vertebrador de la esencia de lo que denominamos «saber enfermero». El cuidar es una ciencia y un arte, requiere un tiempo, una dedicación, conlleva una ética y una estética, presupone un conocimiento teórico y práctico y sobre todo un marco específico donde éste pueda llevarse a cabo.

Sólo a través de una interiorización del cuidado y de una profundización como instrumento (operativo) propio de enfermería nos dará la verdadera proyección y dimensionamiento del modelo formativo profesional que queremos para la aplicación de un adecuado ejercicio profesional.

Desde una perspectiva cultural, el cuidado es un constructo complejo que debe centrarse en la alteridad del sujeto, mediatizando la relación interpersonal que establecemos con el paciente. La nueva cosmovisión del cuidado debe de contemplar los aspectos socioculturales, los valores, las creencias, los modos de vida, las distintas concepciones de salud y enfermedad, en suma... la diversidad cultural. Esta nueva mirada nos puede aportar una visión amplia conducente a la adquisición de nuevas habilidades, al crecimiento personal y a la comprensión del cuidado como «hecho total».

\section{HACIA UN «NUEVO» MARCO CONCEPTUAL}

Enfermería necesita orientarse hacia un marco conceptual que sea inherente a la propia esencia de la disciplina, que no es otra que el cuidado. Desde una perspectiva antropológica, y como profesionales de salud venimos percibiendo desde hace unos años para acá, el surgimiento de una diversidad de posiciones epistemológicas en oposición al paradigma positivista ${ }^{1}$, racional y

\footnotetext{
' Medina Moya, J.L. La investigación enfermera: entre el mito de la racionalidad y la necesidad de un cambio de paradigma. Enf. Clin. 1994; 4(4):7-8.
} 
tecnocrático dominante y todavía vigente en gran parte de la estructura y el marco de actuación de Enfermería. Esto se ha podido constatar en la evolución de los estudios de ATS que hasta 1977 seguian una formación técnica y auxiliar del paradigma positivista (médico) dominante. A partir de esta fecha en la que los estudios de Enfermería se hacen universitarios; los enfermeros adoptarán ese modelo imperante, hasta que posteriormente, a finales de los años ochenta y en adelante, se observa un inusitado interés por otro tipo de metodología (cualitativa y más abierta) centrada más en contextos humanos y sociales en relación al acto de cuidar.

Enfermería es quizás, la única disciplina dentro de las ciencias de la salud que no posee un método propio, unas perspectivas teóricas o marcos de estructuras de análisis de la realidad humana con la que trabaja. Se adolece de una estructura de pensamiento (o de racionalidad) como promulgaba Habermas² (1982), de un paradigma (Kuhn', 1962) o de un marco metatérico (Botha ${ }^{4}$, 1989) alentador y definitorio de nuestro propio marco referencial.

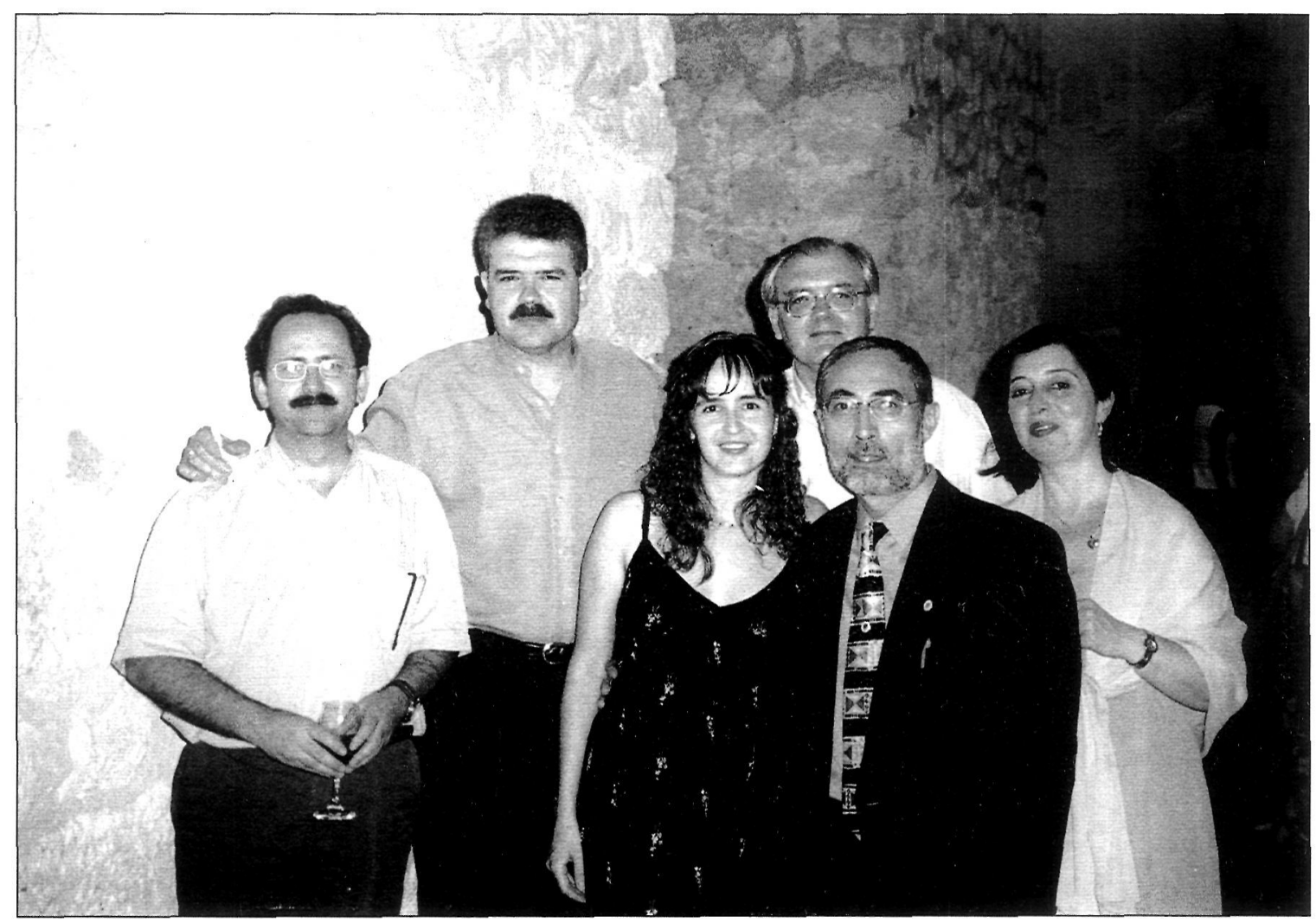

${ }^{2}$ Habermas, J. (1982). Conocimiento e interés. Madrid. Taurus, [lo define como: kel conjunto de ideas, creencias, actitudes y sentimientos, asi como también prácticas que mediatizan la relación del individuo con su entorno»].

'Kuhn, Thomas S. (1986) [orig. 1962]. La estructura de las revoluciones cientificas. México: FCE, [lo define como: «realizaciones cientificas universalmente reconocidas, que durante cierto tiempo proporcionan modelos de problemas y soluciones a una comunidad cientifica», op. cit., p. 3]

+Véase al respecto: Botha, E. Theory development in perspective: role of conceptual frameworks and models in theory development. J. Adv Nursing 1989; 14:49-55. 


\section{Enfermería clásicamente conformada en} torno a la praxis ha crecido y se ha desarrollado ayuna de un marco teórico vertebrador sobre el que apoyarse. Y esa praxis derivada de las artes ha favorecido las técnicas de cuidados sobre los aspectos de reflexión teórica, conformando un divorcio entre lo teórico y lo práxico, que aún hoy se plasma en la actividad que desarrollan los enfermeros en su práctica asistencial hospitalaria y los marcos teorizadores que se enseñan en las escuelas universitarias. Hay que tender a una adecuación de la formación teórica fundamentadora de dicha praxis, que permita una normalización en la formación de nuestros profesionales y que garantice una prestación de los cuidados de forma homogénea y plena. Los aspectos de la reflexión teórica deben permear sobre los aspectos prácticos.

$\mathrm{Y}$ ha sido esa indefinición por ausencia de una fundamentación específica del conocimiento (epistemología), la que ha imposibilitado la construcción de unas categorizaciones teóricas, de unos modelos y de un marco conceptual propio que nos sirviera como referente profesional posibilitando la unicidad de criterios y facilitando la toma de decisiones cualquiera que fuera el ámbito de nuestra actuación.

Personalmente, opino que nos encontramos en una nueva fase «transformadora», «preparadigmática» (aunque algunos lo cuestionen ${ }^{5}$ ) o de «crisis resolutiva de método». En el discurrir enfermero hemos echado en falta, una fase que yo denominaría de "eclosión multiparadigmática»", tal como ha ocurrido en otros países hace unas décadas, que nos ha llevado a una situación de «impasse» y a una indefinición profesional y, a lo que es peor, a no haber sido capaces de dar una imagen nítida a la sociedad de quienes somos y de cuál es nuestro papel en la prestación directa asistencial al paciente.

\section{UNA NUEVA ENFERMERÍA PARA UNA SOCIEDAD CAMBIANTE: UN RETO PARA EL SIGLO XXI}

Nuestra sociedad sufre constantemente fuertes procesos de cambio social. La reorganización de las clases sociales, la aparición de nuevos movimientos, la emergencia de nacionalismos y fundamentalismos de todo tipo, los flujos migratorios, la gran diversidad cultural y étnica (multiculturalismo) que empiezan a darse en nuestra sociedad nos obliga a reorientar nuestra filosofía del cuidado, hacia una identidad nueva que superando retos e incertidumbres y conflictos entre roles interprofesionales, busque la centralidad del cuidado como axioma operativo.

La práctica del cuidado repercute en todos estos aspectos conformadores (personales, familiares, de relación, sociales,..) del ser humano y redimensiona nuestra acción desde una perspectiva humanística y antropológica. Necesitamos de un modelo propio marcadamente humanístico e intercultural con cierta flexibilidad metodológica y basado en la comprensión e interpretación de los hechos individuales de cada sujeto. Un modelo operacional en términos de autonomía personal y como aludía Ivan Illich (al referirse al concepto de salud) con «capacidad de adaptarse a ambientes cambiantes» dentro de una actuación en un contexto social amplio, marcado todo ello y adaptado a los

\footnotetext{
'Meleis, A. (1991) en su libro: Theorethical Nursing. Development \& Progress, 2.a ed. Filadelfia: Lipincott, niega que Enfermería se encuentre en dicha fase, si bien el contexto de su análisis y el marco de su teoría no es el nuestro.

"Esto último se ha venido soslayando en cierta medida con el trabajo coordinado y desmedido de revistas como Cultura de los Cuidados, Revista de Enfermeria y Humanidades, (vol. 1, n. ${ }^{\circ}$ 1, en 1997) y la creación del Seminario de Historia y Antropologia de los Cuidados Enfermeros, adscritos ambos, al Departamento de Enfermería de la Universidad de Alicante. U otras, como Índex de Enfermeria (Granada, 1992) que han abierto sus páginas a aspectos varios del conocimiento antropológico, fenomenológico y teoréticos del cuidado de enfermería.
} 
códigos culturales de un grupo, de su entorno y de su historia. El cuidado es un constructo universal y no entiende de etnias, géneros, edad o grupos sociales y es por ello que el cuidado desde siempre -y cada vez más- tiene una mayor proyección psicosocial y cultural que nuestra profesión debe saber vislumbrar.

Se abren nuevos espacios y Enfermería necesita encontrar el suyo propio de cara al siglo veintiuno. Desde una perspectiva personal, y siguiendo a Marc Augé, tres podrían ser los pilares básicos de esa «nueva enfermeria»: el identitario, el relacional y el histórico. El identitario como aspecto esencial en donde la inmensa mayoría de los profesionales seamos capaces de autorreconocernos; el relacional, entendido como marco específico de actuación conformativa y de interacción personal y el histórico como aspecto de filiación, de reconocimiento en el tiempo como instrumento de toma de conciencia imprescindible para el cambio necesario que postulamos. Es la ausencia de algunos de estos aspectos lo que marcaría, lo que Augé define como los «no lugares $\rangle^{7}$, es decir, la ausencia de una ubicación determinada en el contexto de salud, el estigma de la invisibilidad que conlleva nuestro colectivo en muchos aspectos de nuestro quehacer cotidiano.

Y llegados a este punto de reflexión surgen las eternas preguntas: ¿existe una identidad de colectivo?, ¿cumple enfermeria una labor social necesaria?, ¿cuál es la aportación enfermera?, ¿hay un producto enfermero especifico?, ise percibe por parte de la inmensa mayoría del colectivo la necesidad de la definición de un modelo?, ¿se puede responder a todo esto desde la indefinición actual que nos encontramos?... presiento que las respuestas a todas estas cuestiones pueden ser tan dispares y complejas como los profesionales que integramos este amplio colectivo.
Ante este panorama incierto, me pregunto, como muchos hacen: - icuál es entonces el futuro que nos espera? - El futuro va a depender de nuestra capacidad de adaptación y de cambio a los nuevos tiempos, a las transformaciones que se están dando y que inciden en los ámbitos de la interdisciplinariedad. Creo que los profesionales deberemos estar muy atentos a las «nuevas formas» que demanda la sociedad del colectivo enfermero, y que ese nuevo modelo deberá de inspirarse en el tipo de necesidades detectadas. Desde esta perspectiva, podriamos decir que el futuro será, en cierta medida, el que los profesionales «queramos» que sea.

\section{¿QUÉ APORTA LA ANTROPOLOGÍA A LA ENFERMERIA?}

Se me ha encomendado por la coordinación de esta mesa que hiciera un breve análisis — desde mi doble condición de enfermero y antropólogosobre que puede aportar la Antropología a la Enfermería como disciplina. Y ello supone partir inicialmente de un supuesto básico como es el reconocimiento implícito que hacen compartir al ser humano como objeto de estudio común a ambas disciplinas, si bien es cierto, que desde distintas perspectivas.

Desde mi experiencia, la Antropologia nos ayuda a reinterpretar, entre otros, conceptos básicos como los de salud y enfermedad; dado que lo social y lo cultural tienen cada vez más peso en los aspectos conformacionales de ambos procesos. La cultura es algo inherente al ser humano, y por tanto la identidad cultural debe de formar parte de cualquier paradigma que intente abordar al ser humano desde la prestación del cuidado.

La Antropología puede aportar a la Enfermeria un nuevo marco sistémico de trabajo, en donde los aspectos teóricos y los técnicos pasa-

־Augé, Marc (1995). Hacia una Antropología de los Mundos Contemporáneos. Barcelona: Gedisa. 
rían inexorablemente por la frontera de lo metodológico ${ }^{8}$. La Antropología de los Cuidados como disciplina social intenta comprender al ser humano holisticamente (bio-psico-social) y desde esa perspectiva sólo puede ser idiográfica, particularista y partir de la comprensión" (verstehen) como visión de análisis. Dado que la Enfermería es una disciplina holística, en donde el sujeto es nuestro «laboratorio interno» de experimentación hace que basarse en una metodología que preconize la fragmentación analítica y la pura cuantificación suponga ignorar esa realidad holística y analizar los problemas fuera del contexto en donde surgen. Por ello la Antropología de los Cuidados y la Enfermería deben de partir de esa otra perspectiva conceptual de análisis. Como decía Max Weber: «No son las relaciones reales entre cosas lo que constituye el principio de delimitación de los diferentes campos científicos sino las relaciones conceptuales entre problemas. Sólo allí, donde se aplica un método nuevo a nuevos problemas y donde, por tanto, se descubren nuevas perspectivas nace una ciencia nueva».

¿Hacia dónde va la Enfermeria? hacia una Antropología Enfermera, hacia una Etnoenfermeria, hacia una Enfermería Transcultural... como decíamos, en España hemos echado en falta esa eclosión multiparadigmática que desde distintas visiones hubieran fortalecido epistemologicamente a la Enfermería, pero eso no se ha llegado a darse. La práctica de cuidados, como decíamos en la introducción, es un fenómeno universal, pero las con- ductas, las expresiones y los modelos de cuidados varían de una cultura a otra, y es ahí donde debe incidir enfermería.

A partir de 1994 (Plan 94), en que se modifican en la Universidad Española, los planes de acceso a la licenciatura de Antropología Social y Cultural, permitiendo acceder a la misma tras la finalización del primer ciclo universitario de algunas carreras y los primeros ciclos de otras licenciaturas $^{10}$; supone para algunos profesionales la posibilidad de formación a un nivel de especialización superior, hasta entonces no prevista.

Ello ha permitido el acceso de un gran número de profesionales del ámbito de Enfermería, que ante la ausencia de un segundo y un tercer ciclo propio de enseñanza universitaria han optado por esta modalidad de formación complementaria en aras a una mayor profundización en su formación universitaria, aunque si bien es cierto, que también con otros intereses tan variados como dispares.

Esto ha evidenciado, una vez más, la necesidad de una licenciatura propia de enfermería y el posibilitar el acceso a un tercer ciclo de formación específica enfermera; cuestión ésta, que ha permitido la «salida al mercado docente» de alguna titulación en antropología ofertada como «la licenciatura de Enfermería», sin ser tal y sin presentar un perfil específico de formación enfermera y sin poder facilitar, por tanto, una titulación de licenciatura en enfermería sino en antropología.

Llegados a este punto de inflexión, cabría preguntarse: ¿qué van a aportar en un futuro esta

\footnotetext{
" Entendiendo por metodología, el conjunto de procedimientos según los cuales el científico social extrae información del campo de trabajo y los moviliza en distintos grados de abstracción con el objeto de la producción de conocimientos.

"Esta versión «comprensiva/interpretativa» es compartida también, desde distintas perspectivas, por la Antropología Postmoderna, la Antropología Hermenéutica o la Etnometodología.

10 La O.M. de 22 de diciembre de 1992, posteriormente ampliada por otra de 10 de diciembre de 1993, establecía las titulaciones conducentes a la obtención del título de licenciado en Antropología Social y Cultural. Las diplomaturas y primeros ciclos de licenciatura establecidos, son los siguientes: Diplomatura de Magisterio (en cualquiera de sus especialidades), Enfermeria, Trabajo Social y Educación Social. Y el primer ciclo de las licenciaturas en: Bellas Artes, Filosofía, Historia del Arte, Economía, Administración y Dirección de Empresas, Sociología, Derecho, Ciencias Politicas, Humanidades, Psicología, Pedagogía, Historia, Geografía, Periodismo, Comunicación Audiovisual y Publicidad, Relaciones Públicas y todas las licenciaturas en Filologia.
} 
pléyade de «enfermeros antropólogos» a la Enfermería?, ¿seguirán interesados en acceder los enfermeros si en un futuro próximo se dispone de una licenciatura propia?, ¿de qué forma repercutirá esta formación en el trabajo base de Enfermería?

Y siendo críticos al máximo, cabría plantearse con seriedad: ¿refleja la identidad enfermera y supone este avance «de unos pocos» el avance y el interés de todo el colectivo?, ¿estamos creando asi- metrías o niveles de percepción enfermera que ven la profesión de manera distinta?... o no estaremos en el fondo o infravalorando o renegando de aspectos vertebradores en la prestación del cuidado como es la función asistencial de base.

Las respuestas a éstas y otras preguntas están en nuestras manos y de ellas va a depender en parte la orientación que entre todos seamos capaces de brindar a nuestro colectivo.

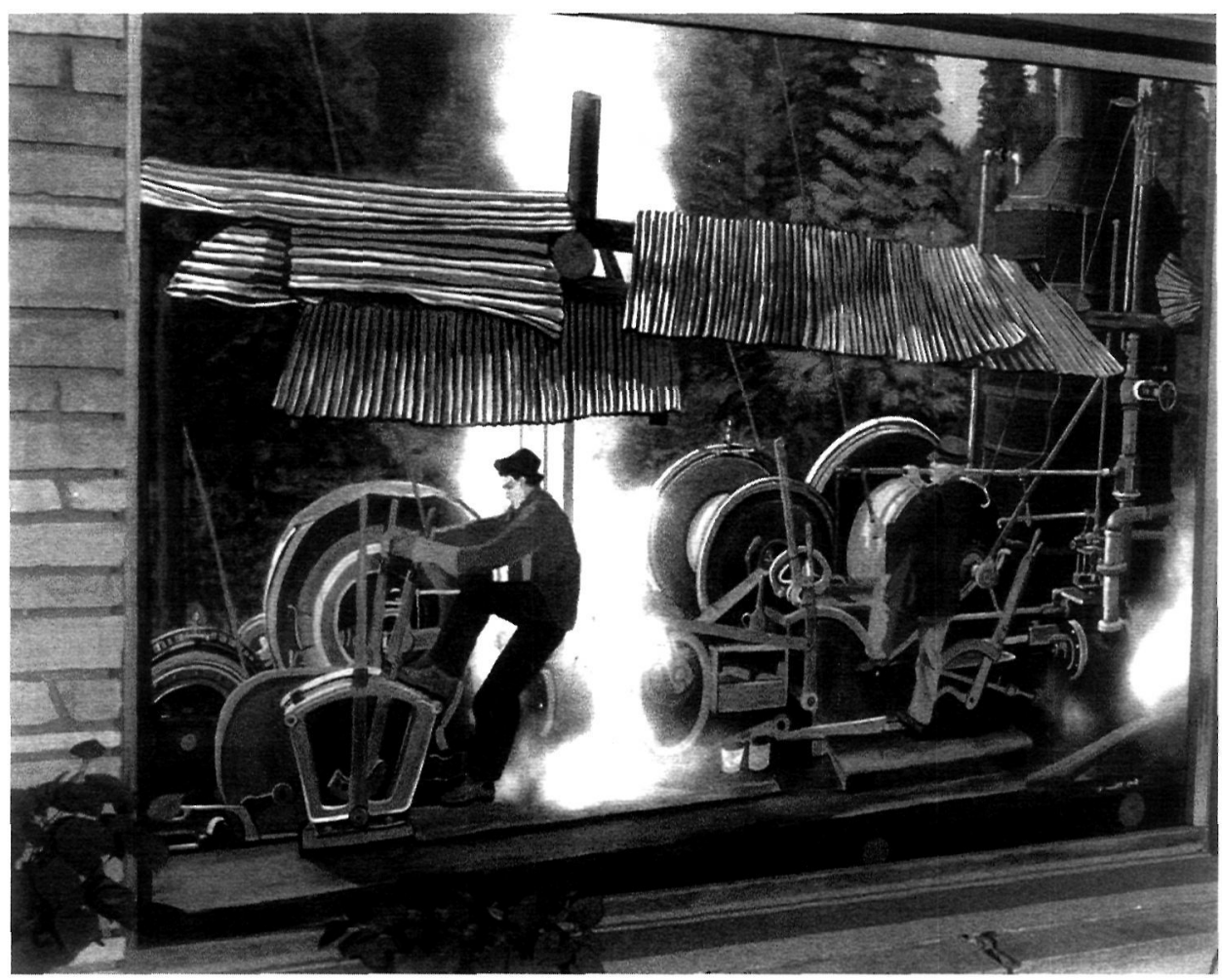

